05

\title{
Упругие и фотоупругие характеристики графеноподобных соединений
}

\author{
() С.Ю. Давыдов
}

Физико-технический институт им. А.Ф. Иофффе РАН, Санкт-Петербург Санкт-Петербургский национальный исследовательский университет информационных технологий, механики и оптики

E-mail: Sergei_Davydov@mail.ru

Поступило в Редакцию 26 сентября 2016 г.

Для 13 графеноподобных соединений IV-IV и III-V получены значения центральной и нецентральной гармонических силовых констант модели Китинга, предложенной ранее автором для описания упругих постоянных графена. Показано, что с ростом ионности соединения относительная роль нецентральных сил уменьшается. Для графена оценено значение ангармонической константы центрального взаимодействия и вычислены зависимости упругих постоянных второго порядка от двумерного давления. Определены значения фотоупругих постоянных.

DOI: $10.21883 /$ PJTF.2017.05.44362.16490

В последние годы интерес к графеноподобным соединениям (ГПС) и структурам на их основе заметно растет [1-3]. Этот интерес связан, в первую очередь, с тем обстоятельством, что такие соединения (за исключением графена, силицена и, возможно, германена) обладают в свободном состоянии запрещенной зоной, шириной которой можно управлять, что весьма удобно для приборных применений. Поэтому естественно, что основное внимание уделяется электронной структуре ГПС, а упругие свойства находятся как бы на периферии исследований. В настоящей работе мы рассмотрим упругие и фотоупругие константы двумерных бинарных соединений IV-IV и III-V с гексагональной структурой.

Необходимо также отметить, что подавляющее число теоретических работ по ГПС выполнены в рамках различных вариантов формализма функционала плотности. Так как, однако, многие характеристики ГПС еще не исследованы экспериментально и, более того, ряд таких соединений до сих пор являются гипотетическими, модельный подход к 
расчету таких характеристик вполне оправдан. Именно такой подход и использован в настоящей работе.

В [4] для описания упругих свойств ГПС была использована модель Китинга [5], в рамках которой упругие постоянные второго порядка $c_{i j}$ имеют вид

$$
c_{11}=\frac{1}{\sqrt{3}}\left(4 \alpha+\beta+18 \frac{\alpha \beta}{4 \alpha+\beta}\right), c_{12}=\frac{1}{\sqrt{3}}\left(4 \alpha+\beta-18 \frac{\alpha \beta}{4 \alpha+\beta}\right),
$$

где гармонические силовые константы $\alpha$ и $\beta$ описывают центральное и нецентральное взаимодействия соответственно. Для определения силовых констант воспользуемся результатами численных расчетов [6]. Пересчет проводился по формулам: $4 \alpha+\beta=\sqrt{3} E / 2(1-\sigma)$, $\alpha \beta=E^{2} / 24\left(1-\sigma^{2}\right)$, где модули Юнга $E=c_{11}\left(1-\sigma^{2}\right)$ и коэффициенты Пуассона $\sigma=c_{12} / c_{11}$ брались из работы [6], где модуль Юнга и коэффициент Пуассона обозначены соответственно как $C$ и v. Результаты расчетов констант $\alpha$ и $\beta$ сведены в таблицу. Из таблицы следует, что наибольшие значения констант $\alpha$ и $\beta$ соответствуют графену и гексагональному нитриду бора. Отношение $\beta / \alpha$ характеризует „удельный вес“ нецентрального взаимодействия по отношению к центральному. Из рис. 1 следует, что с ростом ионности $f_{i}$ по Филлипсу [7] отношение $\beta / \alpha$ убывает. Та же тенденция наблюдается и для тетраэдрических полупроводников при переходе от алмаза к соединениям III-V и II-VI [8].

Перейдем теперь к упругим постоянным третьего порядка $c_{i j k}$, выражения для которых были получены нами в работе [9]:

$$
\begin{aligned}
& c_{111}=(16 \gamma / \sqrt{3})\left[(1.5-\zeta)^{3}+4 \xi^{3}\right], \\
& c_{222}=(16 \gamma / \sqrt{3})\left[(0.5+\zeta)^{3}+4(1-\xi)^{3}\right], \\
& c_{112}=(16 \gamma / 3 \sqrt{3})\left[(1.5-\xi)^{2}(0.5+\xi)+4 \xi^{2}(1-\zeta)\right],
\end{aligned}
$$

где $\gamma$ - ангармоническая силовая константа центрального взаимодействия и $\xi=(2 \alpha-\beta) /(4 \alpha+\beta)$ - параметр внутренних смещений Клейнмана.

Для дальнейших оценок положим для графена $\alpha=\beta$, что с хорошей точность соответствует полученным здесь результатам (см. таблицу и рис. 1 ), а также оценкам работы [10], где $\alpha \approx 77 \mathrm{~N} / \mathrm{m}$ и $\beta \approx 69 \mathrm{~N} / \mathrm{m}$. То-

Письма в ЖТФ, 2017, том 43, вып. 5 
Таблица 1. Силовые константы $\alpha$ и $\beta$ (в ед. $\mathrm{N} / \mathrm{m}$ ), ионность соединения по Филлипсу $f_{i}$, коэффициент Пуассона $\sigma[6]$, диэлектрические электронная восприимчивость $\chi_{1}^{e l}$ и высокочастотная проницаемость $\varepsilon_{\infty}($ при $\bar{\gamma}=1)$ графеноподобных соединений IV-IV и III-V

\begin{tabular}{c|c|c|c|c|c|c}
\hline Соединение & $\mathrm{Gr}$ & $\mathrm{SI}$ & $\mathrm{Gm}$ & $\mathrm{SiC}$ & $\mathrm{GeC}$ & $\mathrm{GeSi}$ \\
\hline$\alpha$ & 69 & 17.0 & 13.5 & 43 & 33 & 16 \\
$\beta$ & 70 & 9.5 & 8.0 & 29 & 29 & 9.6 \\
$f_{i}$ & 0 & 0 & 0 & 0.09 & 0.09 & 0 \\
$\sigma$ & 0.16 & 0.30 & 0.33 & 0.29 & 0.33 & 0.32 \\
$\chi_{1}^{e l}$ & 0.44 & 0.49 & 0.40 & 0.44 & 0.44 & 0.45 \\
$\varepsilon_{\infty}$ & 6.53 & 7.16 & 6.06 & 6.47 & 6.59 & 6.60
\end{tabular}

\begin{tabular}{c|c|c|c|c|c|c|c}
\hline Соединение & BN & BP & BAs & AlN & GaN & GaP & GaAs \\
\hline$\alpha$ & 60 & 35 & 31 & 40 & 42 & 17 & 14 \\
$\beta$ & 52 & 23 & 21 & 18 & 16 & 9.6 & 7.8 \\
$f_{i}$ & 0.14 & 0.06 & 0.03 & 0.43 & 0.45 & 0.30 & 0.27 \\
$\sigma$ & 0.21 & 0.28 & 0.29 & 0.46 & 0.48 & 0.35 & 0.35 \\
$\chi_{1}^{e l}$ & 0.36 & 0.50 & 0.50 & 0.37 & 0.38 & 0.53 & 0.55 \\
$\varepsilon_{\infty}$ & 5.47 & 7.23 & 7.23 & 5.62 & 5.78 & 7.69 & 7.89
\end{tabular}

Примечание: $\mathrm{Gr}$ - графен, SI - силицен, Gm - германен.

гда $\xi=1 / 5$ и вместо (2) находим $c_{111} \approx 21 \gamma, c_{222} \approx 22 \gamma, c_{112} \approx 4 \gamma$. Воспользуемся теперь результатами численных расчетов [11], где получены следующие значения: $c_{111}=-2832, c_{222}=-2684$ и $c_{112}=-390 \mathrm{~N} / \mathrm{m}$. Сопоставляя средние величины $c_{111}$ и $c_{222}$, получим $\gamma=-129 \mathrm{~N} / \mathrm{m}$, так что $c_{111}=-2731, c_{222}=-2855$ и $c_{112}=-517 \mathrm{~N} / \mathrm{m}$ (отметим, что в соответствии с [11] и формулами (1) имеем $\alpha \approx 71 \mathrm{~N} / \mathrm{m}, \beta \approx 66 \mathrm{~N} / \mathrm{m}$ и $\beta / \alpha \approx 0.92$, так что принятое нами упрощение $\alpha=\beta$ работает). Таким образом, по порядку величины упругие постоянные $c_{111}$ и $c_{222}$ достаточно хорошо описываются выражением (2) с той, однако, поправкой, что у нас $\left|c_{111}\right|<\left|c_{222}\right|$, тогда как в [11] имеет место обратное неравенство. Что касается величины упругой постоянной $\left|c_{112}\right|$, то полученное нами значение приблизительно на треть превосходит результат работы [11]. С другой стороны, по данным [12] $c_{111}=-2725, c_{222}=-2523$, $c_{112}=-591 \mathrm{~N} / \mathrm{m}$, откуда $\gamma=-123 \mathrm{~N} / \mathrm{m}$, так что формулы (2) дают

Письма в ЖТФ, 2017, том 43, вып. 5 


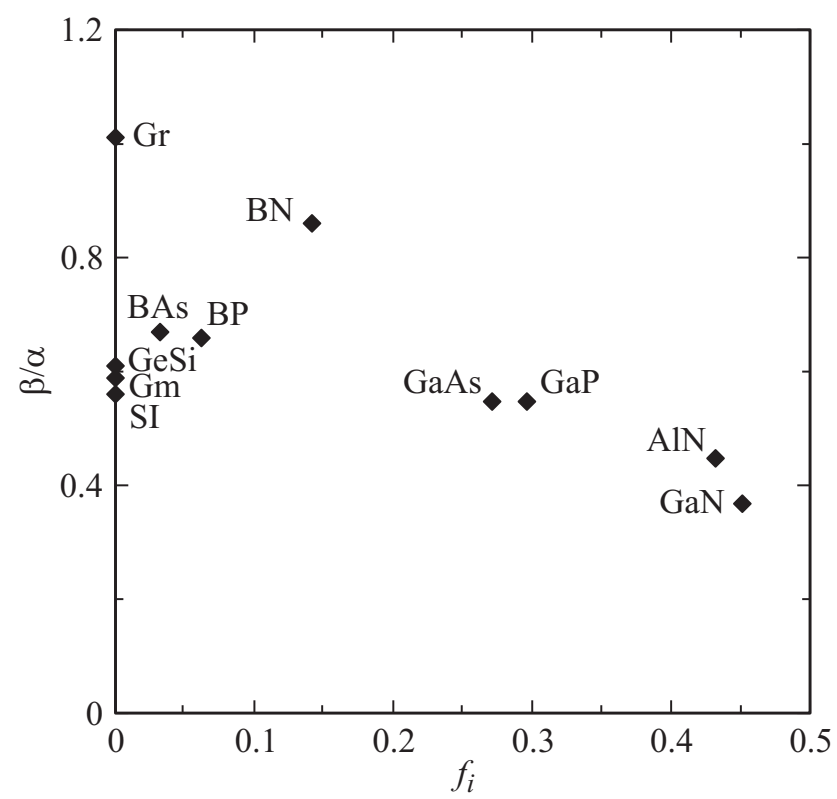

Рис. 1. Отношение нецентральной и центральной силовых констант $\beta / \alpha$ в функции от ионности $f_{i}$ по Филлипсу.

$c_{111}=-2532, c_{222}=-2716$ и $c_{112}=-492 \mathrm{~N} / \mathrm{m}$. Следовательно, в данном случае наше значение $\left|c_{112}\right|$ на $18 \%$ меньше, чем полученное в [12] значение $\left|c_{112}\right|$.

Воспользовавшись результатами работ $[10,13]$, легко показать, что под действием внешнего двумерного давления $P$ относительные изменения упругих постоянных второго порядка $\eta_{i j}=\partial c_{i j}(P) / \partial P$ есть

$$
\eta_{11}=-\frac{c_{111}+c_{112}}{c_{11}+c_{12}}, \quad \eta_{22}=-\frac{c_{222}}{c_{11}+c_{12}}, \quad \eta_{12}=-\frac{c_{112}}{c_{11}+c_{12}} .
$$

Воспользовавшись данными работы [11], для графена получаем $\eta_{11} \approx 7.94, \eta_{22} \approx 6.61$ и $\eta_{12} \approx 0.96$. Отметим, что значения $\eta_{11}$ и $\eta_{22}$ приблизительно в полтора раза превышают значения $\eta_{11}^{\prime}$ и $\eta_{12}^{\prime}$ для объемных образцов кремния и германия, тогда как $\eta_{12} \sim \eta_{14}^{\prime}$.

Перейдем теперь к фотоупругим постоянным $p_{i j}$. Насколько известно автору, выражения для $p_{i j}$ двумерной гексагональной структуры

Письма в ЖТФ, 2017, том 43, вып. 5 
впервые получены в работе [14]. Величины (модули) $p_{i j}$ даются формулами

$$
p_{11}=\frac{\varepsilon_{\infty}-1}{2 \varepsilon_{\infty}^{2}} \frac{1}{1+\sigma}, \quad p_{12}=\sigma p_{11},
$$

где $\varepsilon_{\infty}=1+4 \pi \chi_{1}^{e}-$ высокочастотная диэлектрическая проницаемость, $\chi_{1}^{e}$ - электронная диэлектрическая восприимчивость первого порядка. Воспользовавшись методом связывающих орбиталей Харрисона [15], можно показать (см. [16] и ссылки, приведенные там), что

$$
\chi_{1}^{e l}=\frac{1}{\sqrt{3}} \frac{(e \bar{\gamma})^{2} \alpha_{c}^{3} g^{(1)}}{V_{2} a}, \quad g^{(1)}=1-2 \alpha_{c}^{2}\left(1-5 \alpha_{p}^{2}\right) \frac{V_{1}^{2}}{V_{2}^{2}} .
$$

Здесь $V_{2}$-ковалентная энергия $\sigma$-связи $s p^{2}$-орбиталей ближайших соседей, расстояние между которыми равно $a, V_{1}$ - энергия металлизации, $\alpha_{c}\left(\alpha_{p}\right)$ - ковалентность (полярность) связи, $e-$ заряд электрона, $\bar{\gamma} \sim 1$ - безразмерный параметр (см. подробнее в $[15,16]$ ). В таблице приведены значения $\chi_{1}^{e l}$ и $\varepsilon_{\infty}$, вычисленные, как и в [17], с учетом точных, а не приближенных значений (как в [16]) энергии металлизации $V_{1}$ (точное значение $V_{1}$ превышает приближенное в $2 / \sqrt{3}$ раза $[16,17])$. При этом мы полагали $\bar{\gamma}=1$.

Результаты расчетов фотоупругих постоянных $p_{11}$ и $p_{12}$ представлены на рис. 2. Максимальными значениями $p_{11}$ обладают гексагональный нитрид бора и графен, что обусловлено малыми коэффициентами Пуассона $\sigma$ (см. таблицу). По той же причине значение $p_{12}$ для графена минимально. Полученное нами для графена значение $p_{11} \approx 5.52 \cdot 10^{-2}$ вполне удовлетворительно согласуется с результатами работы [14], тогда как $p_{12} \approx 0.88 \cdot 10^{-2}$ значительно меньше $p_{12}$ из [14]. Последнее, на наш взгляд, обусловлено крайне завышенным значением коэффициента Пуассона $\sigma=c_{12} / c_{11} \approx 0.62$, использованным в [14] (см. ссылку [11] в [14]). Отметим, что величины $p_{i j}$ для ГПС на порядок меньше, чем для объемных кристаллов (см., например, [18]).

Обсудим упрощения, использованные в настоящей работе. Вопервых, при описании упругих постоянных ограничиваемся взаимодействием ближайших соседей и игнорируем дальнодействующее межионное кулоновское взаимодействие, возникающее в гетерополярных ГПС. В принципе, учет такого взаимодействия несложен (см., например, работу [8] по упругости объемных полупроводников). При этом, однако, приходится вводить дополнительный параметр - поперечный

Письма в ЖТФ, 2017, том 43, вып. 5 


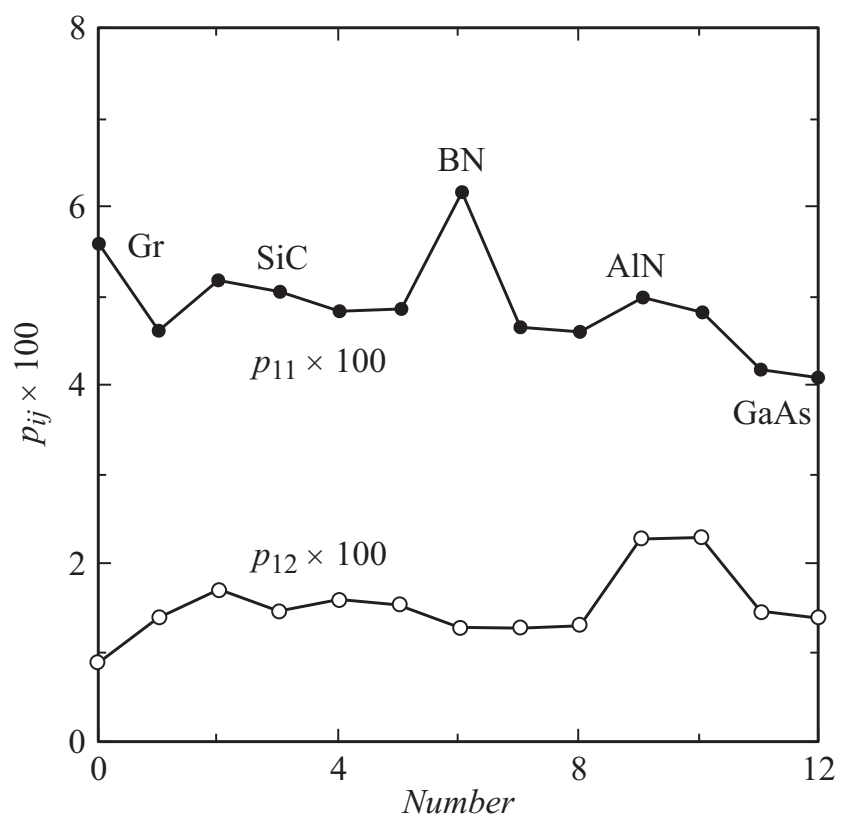

Pис. 2. Значения упругооптических констант $p_{i j}$. Нумерация: $O-\mathrm{Cr}$ (графен), 1 - $\mathrm{SI}$ (силицен), $2-\mathrm{Gm}$ (германен), $3-\mathrm{SiC}, 4-\mathrm{GeC}, 5-\mathrm{GeSi}, 6-\mathrm{BN}$, $7-\mathrm{BP}, 8-\mathrm{BaS}, 9-\mathrm{AlN}, 10-\mathrm{GaN}, 11-\mathrm{GaP}, 12-\mathrm{GaAs}$.

динамический заряд ионов, ответственный за расщепление оптических продольной и поперечной мод в точке Г (см. [17]). Так как ионность рассмотренных нами соединений сравнительно невелика $\left(f_{i}<0.5\right)$, считаем допустимым игнорирование явного учета кулоновского взаимодействия. Здесь имеется определенная аналогия с описанием электронной структуры твердых тел в рамках приближения сильной связи, где дальнодействие явно не учитывается.

Далее, здесь, как и в [9], нами учтена только одна ангармоническая силовая константа $\gamma$, тогда как в работе [13] дополнительно учитываются две нецентральные константы. Не исключено, что, учтя ангармонизм нецентрального взаимодействия, получим для графена $\left|c_{111}\right|>\left|c_{222}\right|$. Однако в отсутствие данных эксперимента решили ограничиться только константой $\gamma$.

Письма в ЖТФ, 2017, том 43, вып. 5 
И, наконец, для всех рассматриваемых ГПС положили учитывающий поправки на локальное поле масштабный фактор $\bar{\gamma}=1$. Для объемных полупроводников в работе [19] (см. также рис. 3.1 в [15]) достаточно убедительно продемонстрировано, что с качественной точки зрения это вполне приемлемо.

Таким образом, в отсутствие соответствующих экспериментальных данных сделанные упрощения представляются нам оправданными.

\section{Список литературы}

[1] Geim A.K., Grigorieva I.V. // Nature. 2013. V. 499. P. 419-425.

[2] Tong C.-J., Zhang H., Zhang Y.-N. et al. // J. Mater. Chem. A. 2014. V. 2. P. 17971-17978.

[3] Антонова И.В. // ФТП. 2016. Т. 50. В. 1. С. 67-82.

[4] Давыдов С.Ю. // ФТТ. 2010. Т. 52. В. 4. С. 756-758.

[5] Keating P.N. // Phys. Rev. 1966. V. 145. N 2. P. 637-645.

[6] Sahin H., Cahangirov S., Topsakal M. et al. // Phys. Rev. B. 2009. V. 80. P. 155453.

[7] Phillips J.C. // Rev. Mod. Phys. 1970. V. 42. N 3. P. 317-356.

[8] Martin R.M. // Phys. Rev. B. 1970. V. 1. N 10. P. 4005-4010.

[9] Давыдов С.Ю. // ФТТ. 2011. Т. 53. В. 3. С. 617-619.

[10] Давыдов С.Ю., Посредник О.В. // ФТТ. 2015. Т. 57. В. 4. С. 819-824.

[11] Wang R, Wang S., Wu X., Liang X. // Physica B. 2010. V. 405. N 16. P. 35013506.

[12] Cadelano E., Palla P.L., Giordano S., Colombo L. // Phys. Rev. Lett. 2009. V. 102. P. 235502.

[13] Keating P.N. // Phys. Rev. 1966. V. 149. N 2. P. 649-658.

[14] Браже Р.А., Кочаев А.И., Мебтахутдинов Р.М. // ФТТ. 2017. Т. 59. В. 2. C. 334-337.

[15] Давыдов С.Ю., Посредник О.В. Метод связывающих орбиталей в теории полупроводников: Учеб. пособие. СПб.: Изд-во СПбГЭТУ „ЛЭТИ“, 2007. 96 c. twirpx.com/file/1014608/

[16] Давыдов С.Ю. // ФТП. 2013. Т. 47. В. 8. С. 1065-1070.

[17] Давыдов С.Ю. // ФТТ. 2016. Т. 58. В. 2. С. 392-400.

[18] Давыдов С.Ю. // ФТП. 2002. Т. 36. В. 1. С. 45-48.

[19] Давыдов С.Ю., Леонов Е.И. // ФТТ. 1987. Т. 29. В. 10. С. 2890-2893.

Письма в ЖТФ, 2017, том 43, вып. 5 